Research Article

\title{
Numerical Model of Plastic Deformation on Cracked Surface under Repeated Pneumatic Impact
}

\author{
Zhiyuan YuanZhou, Bohai Ji iD, Tong Sun, and Zhi Ye \\ College of Civil and Transportation Engineering, Hohai University, Nanjing 210098, China \\ Correspondence should be addressed to Bohai Ji; bhji@hhu.edu.cn
}

Received 26 March 2018; Revised 20 August 2018; Accepted 9 September 2018; Published 30 September 2018

Academic Editor: Aniello Riccio

Copyright (C) 2018 Zhiyuan YuanZhou et al. This is an open access article distributed under the Creative Commons Attribution License, which permits unrestricted use, distribution, and reproduction in any medium, provided the original work is properly cited.

Crack-closure treatment through repeated impact is supposed to be an effective, economical way to extend the fatigue remaining life for fatigue-affected structures with less damage. The material plastic behavior under repeated impact between chisel and material bodies was investigated, for the purpose of describing the crack-closure effect. A numerical model was established associating the impact depth with the number of impact times and device parameters, which is also proved by experiments. The material properties, after repeated impact, was determined via metallographic analysis and microhardness tests, indicating that the material will be hardened within a small affected zone due to the grain refinement caused by repeated impact. The finite-element method was used, as a supplementary approach, to investigate the relationship between impact depth and horizontal deformation on the cracked surface. A one-to-one linear relation was obtained, even under the different chisel tip parameters. Therefore, the contact of fracture surface, i.e. crack closure, can be predicted with the help of this numerical model and the aforementioned relationship.

\section{Introduction}

Fatigue fractures have long been reported: Schütz [1] reviewed the history of fatigue damage from 1837 to 1994, and the contributions to the further development of fatigue knowledge were also described. The purpose of conducting fatigue research can be mainly divided into two aspects: one is to reveal the mechanism driving fatigue, and the other is to explore repair methods thereafter.

The topic of fatigue crack repairing and retrofitting has received great attention in China [2], since many cases of fatigue damage in orthotropic steel bridge decks have been reported in the last 10 years. The fatigue crack will appear where there is a high stress concentration, due to low welding profile, residual tensile stress [3], etc. The longer fatigue cracks are usually repaired and retrofitted after investigation of the cracking, while shorter cracks are left untreated, due to the high cost and time-consuming. Common approaches, such as hole-drilling [4] and rewelding [5], are useful for crack repair and retrofitting; however, these methods will also cause irrecoverable damage; therefore, a new technique causing no, or less, damage is needed.

In the 1990s, the repair of fatigue cracks by hammer impacts was proposed by Fisher and Dexter [6], and some similar investigations were carried out in later research [7], showing that the fatigue life was extended. Based on hammer impact repair techniques, retrofitting by closing the crack surface was developed $[8,9]$. This technique is called the impact crack-closure retrofit (ICR), using an air-impact tool and a chisel. During the process, repeated impacts are introduced near the edge of the crack surface, and contact across fracture surfaces ensued (Figure 1), resulting in the crack-closure effect. Numerous ongoing research studies have been addressed for the evaluation of the ICR technique by its original developers $[10,11]$ and the current authors [12-14], indicating that the fatigue remaining life was extended by this crack-closure effect. Moreover, it provides an economical and environmentally friendly way to improve the load-carrying capacity or extend the service life of structurally deficient members with no, or less, damage. 


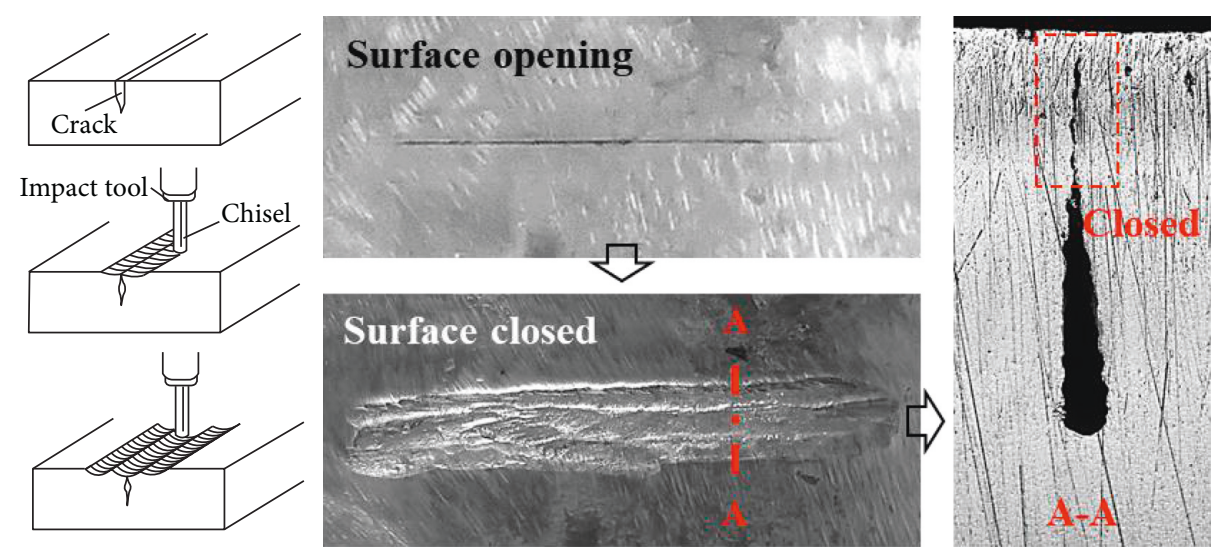

Figure 1: Closed crack surface after repeated impact.

Current investigations of crack-closure treatment are mainly focused on the technical effect, which contributes to improving the fatigue performance of cracked structural members. The discussions of the basic technical effects on material properties, and plastic deformation on the crack surface, remain unclear. In addition, the device parameters used for the impact process are difficult to select due to the unknown relationship between crack-closure behavior and impact parameters, which motivated this research.

In this paper, theoretical and experimental approaches were used in an assessment of the repeated impact process. The material properties, after multiple impacts, were investigated, and the plastic deformation on the cracked surface was analysed. An approximate relationship illustrating the plastic deformation and impact parameters was proposed.

\section{Numerical Model of Cumulative Plastic Deformation}

The process of crack-closure treatment can be approximately considered as a repeated impact process between the chisel and the target material, as shown in Figure 2. The plastic deformation occurs near the surface because of the accumulation of impact energy [15], causing the irrecoverable dislocation of material grains. The impact depth $S$, i.e., plastic deformation, under a single-impact can be calculated as [16]

$$
S=K \sigma_{0}^{2} e^{-\beta / f}
$$

where $K$ and $\beta$ are constants for the material, $\sigma_{0}$ is the mean impact stress, and $f$ is the impact frequency. For steel, the value of $K$ is about $4.736 \times 10^{-8}$ and that of $\beta$ is about 0.36725 .

Since the material strain hardens under repeated impact, this affects the calculated results, and so the strain-hardening effect has to be considered [17]:

$$
S_{N}=S_{N-1} q
$$

where $N$ is the number of impacts, and $q$ is the coefficient of plastic deformation attenuation $(q=0.999745$ for steel).
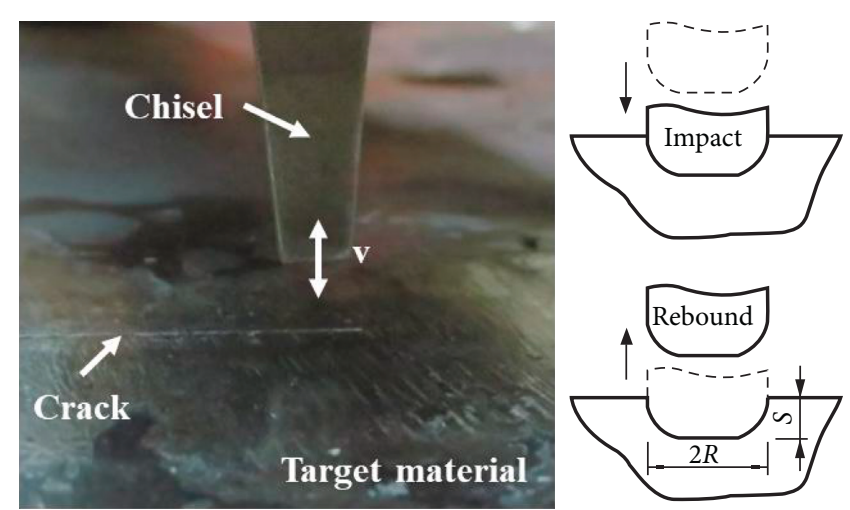

Figure 2: Repeated impact process.

Therefore, the total impact depth $S_{\text {total }}$, i.e., the vertical cumulative plastic deformation (V.CPD), can be written as

$$
S_{\text {total }}=\int_{1}^{N} S_{i}=K \sigma_{0}^{2} e^{-\beta / f} \int_{1}^{N} q^{i-1}=K \sigma_{0}^{2} e^{-\beta / f} \frac{1-q^{N}}{1-q} .
$$

Therefore, the relation between vertical cumulative plastic deformation and number of impacts can be established, only when the mean value of impact stress, $\sigma_{0}$, is obtained. To determine this value, the mean impact force $\bar{F}$ needs to be figured out first, so that the $\sigma_{0}$ can be figured out based on the equation, i.e., $\sigma_{0}=F / A$, where $A$ is the impact size which can be measured directly.

Since the target material was static compared with the motion of the chisel, and the relationship between the chisel impact speed $v$ and impact force $F$ can be given based on the law of conservation of momentum and Newton's second law:

$$
\frac{1}{2} m v^{2}=\int_{0}^{S_{1}} F d S,
$$

where the $m$ is the mass of the chisel, and $v$ is the chisel impact speed which can be determined from:

$$
v=\frac{2 l}{t}=2 l f
$$

where $l$ is the stroke of impact air tool, $t$ is time which is related with the impact frequency. Since each impact time is 
very short when compared with the duration of the entire impact process, the impact force $F$ can be estimated by Hertz contact theory [18] under quasistatic conditions:

$$
F=n S^{3 / 2},
$$

where $n$ is the coefficient, taking the following form:

$$
\begin{aligned}
n & =\frac{4 \sqrt{R}}{3 \pi\left(k_{1}+k_{2}\right)}, \\
k_{1} & =\frac{1-\mu_{1}^{2}}{\pi E_{1}}, \\
k_{2} & =\frac{1-\mu_{2}^{2}}{\pi E_{2}},
\end{aligned}
$$

where $R$ is the radius of the chisel impact area which can be estimated according to the chisel tip size; $E_{1}, \mu_{1}$, and $E_{2}, \mu_{2}$ are the Young's modulus and Poisson ratios of the chisel and target material, respectively. Therefore, the impact force can be obtained by integrating Equation (4):

$$
F=n\left(\frac{5 m}{4 n} v^{2}\right)^{3 / 5} \text {. }
$$

However, the impact force calculated using Equation (7) is the maximum value at one moment, and it will change during the impact process. The mean impact force is the main factor affecting the plastic deformation. The relationship between the mean and maximum impact force is given by

$$
\frac{F}{\bar{F}}=\frac{1.84\left(1+1 / \gamma^{0.2}\right)}{1+\gamma}
$$

where $\bar{F}$ is the mean impact force, and $\gamma$ is the material recovery coefficient ( 0.6 for steel). Thus, the expression of mean impact force is as follows:

$$
\bar{F}=\frac{m(1+\gamma) v^{1.2}}{1.47(5 m / 4 m)^{0.4}\left(1+1 / \gamma^{0.2}\right)} .
$$

Therefore, the model of cumulative plastic deformation is established based on the parameters of impact device, and the plastic deformation can be controlled by adjusting reasonable impact parameters if necessary.

\section{Experimental Investigation}

3.1. Experimental Detail. The samples for experiment were the rectangular blocks cutting from a piece of steel plate with the material of Q345qD according to Chinese specifications. All the sample size was well controlled by wire electrical discharge machining, as shown in Figure 3. The samples were divided into two groups for different experimental purposes. The S1 group was used for the verification purpose of the numerical model where the impact occurred in the middle, while the S2 group was tested to determine the relation of plastic deformation at the edge of crack surface.

Since there is no crack prefabricated in the sample, a simplified approach was then performed, as illustrated in Figure 4. Before cracking occurs, the hypothetical surface

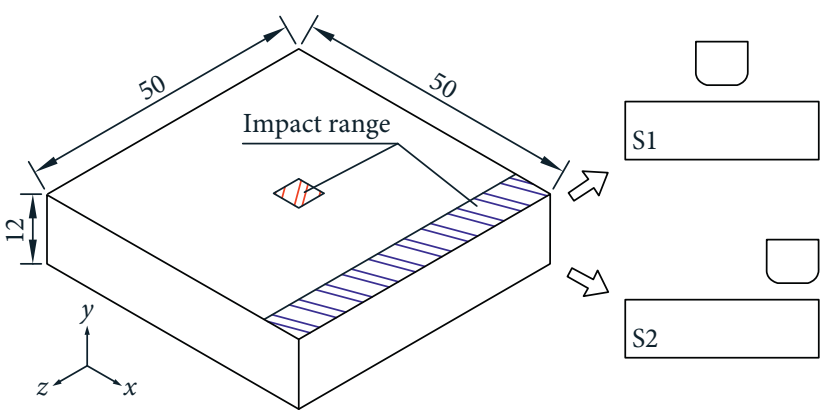

FIGURE 3: Samples for experiment (unit: mm).

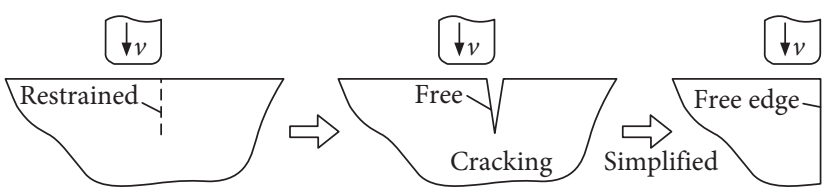

FIgURE 4: Cracked surface.

was restrained by surrounding materials, while it becomes free to deform upon cracking. The behavior of crack surface deformation can be approximately simulated by the edge impact on the target material. Through this simplification, the value of plastic deformation on $X$ and $Y$ directions could be measured directly.

To verify the reasonability of the numerical model provided according to Equation (3), the S1 samples were then tested under different impact durations, i.e., $30 \mathrm{~s}, 60 \mathrm{~s}, 90$, and $120 \mathrm{~s}$, corresponding to impact times of $2700,5400,8100$, and 10800 , respectively. The devices used for repeated pneumatic impact are a portable air compressor, a handheld impact air tool, and a chisel, as shown in Figure 5. The chisel tip was burnished to a flat square surface of $5 \mathrm{~mm} \times 5 \mathrm{~mm}$ with its corner rounded $(r=0.5 \mathrm{~mm})$, because the $5 \mathrm{~mm} \times 5 \mathrm{~mm}$ chisel size, or similar size, close to $5 \mathrm{~mm} \times 5 \mathrm{~mm}$ chisel shape is commonly used in actual treatment. Other parameters are listed in Table 1.

During the impact process, the impact tool was held by the operator, and the chisel was put at the height of $5 \mathrm{~mm}$ considering the stroke range. The impact depth, i.e., the V. $\mathrm{CPD}$, was measured by the depth indicator with the minimum range of $10 \mu \mathrm{m}$. The horizontal cumulative plastic deformation (H.CPD) was obtained by deltas calculation between total deformation size and original size. Since the rough surface occurred after repeated impact, each value was measured for three times, and the average value was taken as the final data.

After necessary measurement, some samples were further cut out for microscale analysis with a similar shape. Part of the sample surfaces were processed by polishing and nitaletching with $4 \% \mathrm{v} / \mathrm{v}$. Three reference surfaces were selected and numbered from I to III, as shown in Figure 6. The metallurgical microscope (Olympus BX51M) was used to capture the grains under the magnification of 200 times, and each image was clipped to $200 \mu \mathrm{m} \times 200 \mu \mathrm{m}$ size for evaluation of grain size. Then, the microhardness test was carried out to measure the material Vicker hardness, as this was 

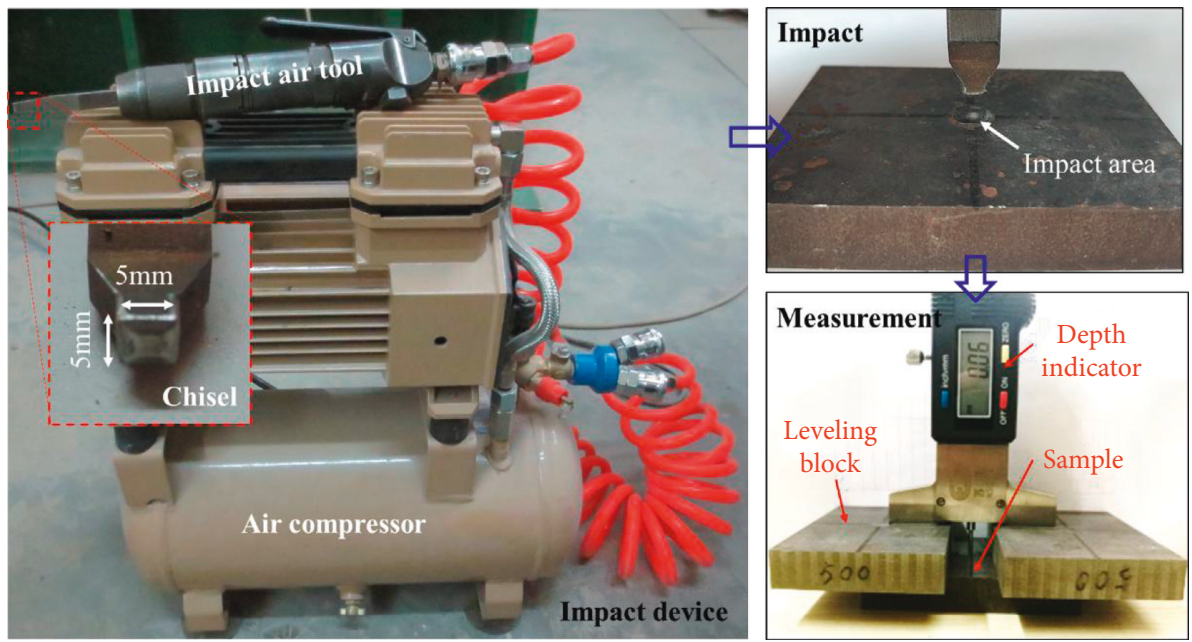

Figure 5: Testing set-up.

Table 1: Parameters.

\begin{tabular}{lcccccc}
\hline & Impact air tool & & \multicolumn{2}{c}{ Chisel } & \multicolumn{2}{c}{ Target material (Q345qD) } \\
$f(\mathrm{~Hz})$ & Stroke $(\mathrm{mm})$ & $m(\mathrm{Kg})$ & $E(\mathrm{GPa})$ & $\mu$ & $E(\mathrm{GPa})$ & $\mu$ \\
\hline 90 & 6 & 0.1 & 206 & 0.3 & 206 & 0.3 \\
\hline
\end{tabular}

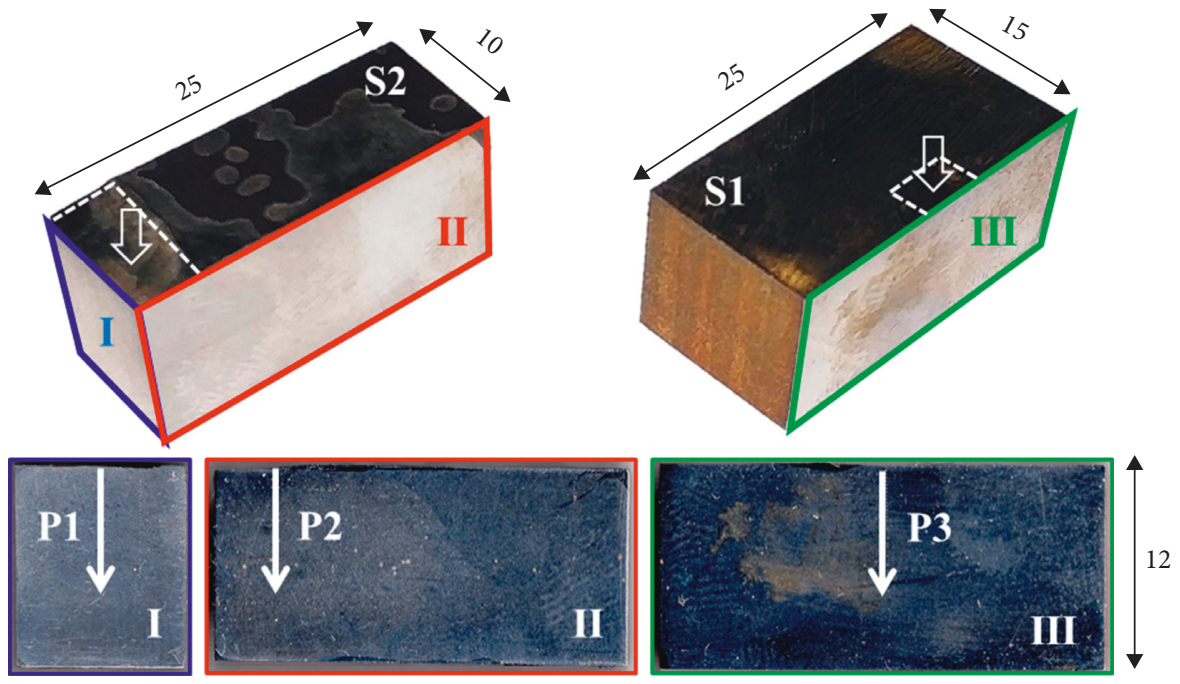

FIgURE 6: Samples cut out (unit: mm).

deemed to be a comprehensive index reflecting the material strength, toughness, internal residual stress field, etc. The microhardness test force is $200 \mathrm{gf}$, and the loading time is 15 s. Figure 6 also shows the microhardness test paths, and they are all selected so as to be underneath the impacted area.

3.2. Verification of Numerical Model. The relationship between V.CPD (impact depth) and number of impacts $N$ was then illustrated in Figure 7, as well as the numerical model predicted by Equation (3). It is found that the V.CPD increases rapidly during the early stage, while it gradually reaches a plateau with increasing number of impacts, because the effect on the plastic deformation due to material strain hardening is increasing in the later stage, as coefficient $q$ predicted in Equation (2). The mean test data under different impact times also describe the tendency of V.CPD and agree well with the solutions found during the early stages predicted by Equation (3). Therefore, Equation (3) is able to predict the V.CPD for common steel under repeated pneumatic impact with good accuracy, and the relative parameters for crack-closure treatment can be predicted. As for other materials, the relative coefficients are necessary to investigate by basic material test, to modify the solutions.

3.3. Grain Size Evaluation. Figure 8 shows the grain images captured by microscope. The images presenting the impact 


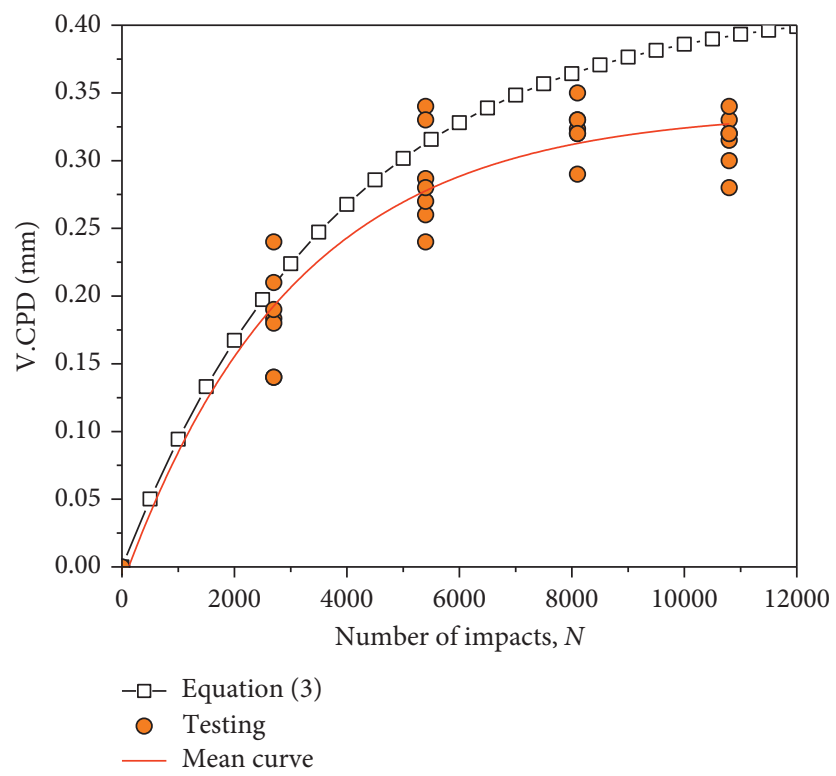

Figure 7: Cumulative plastic deformation.

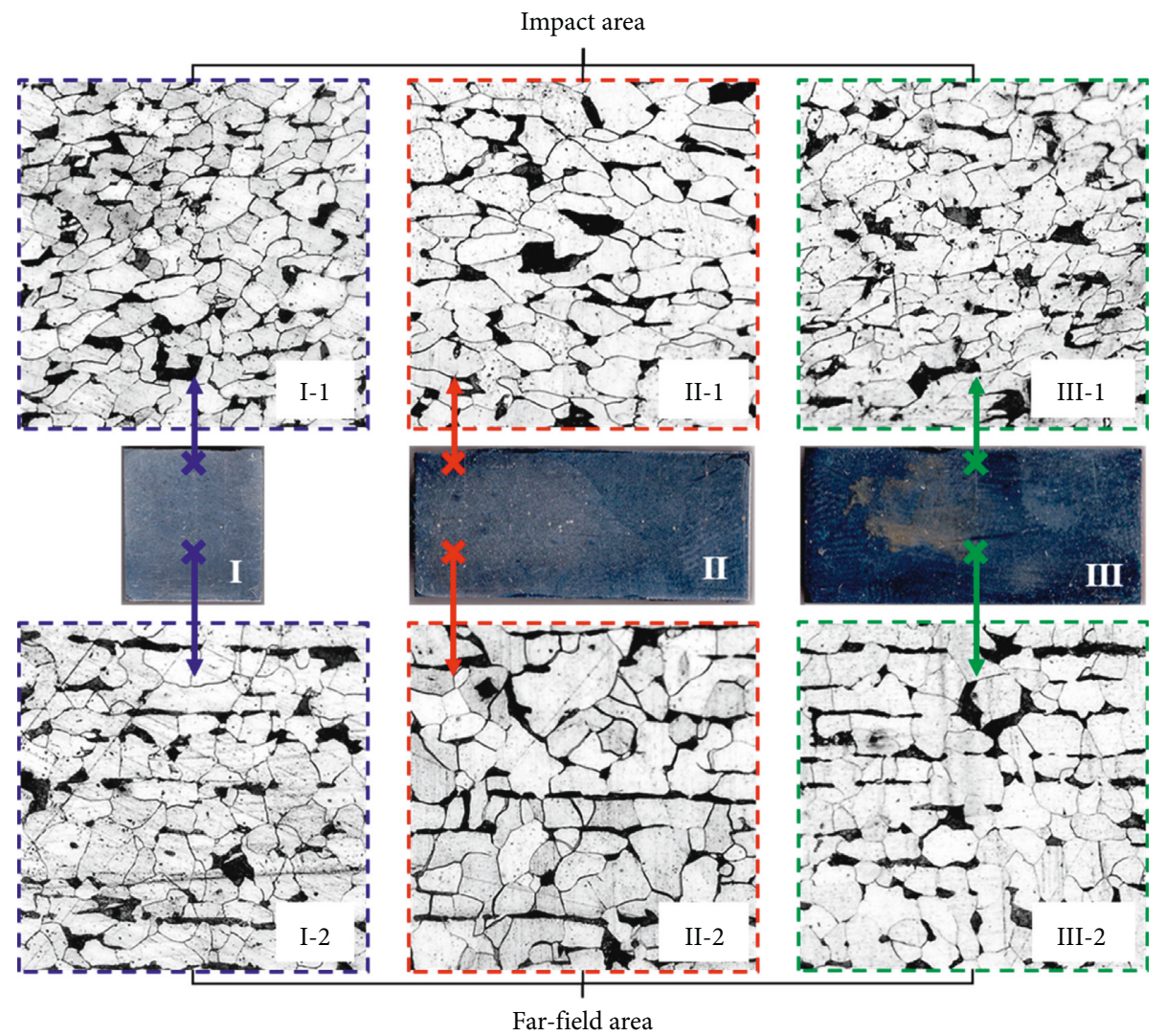

Figure 8: Sample metallographs $(\times 200)$.

area are numbered from I-1 to III-1, and others numbered from I- 2 to III- 2 are captured from the far-field area. It turns out that the average sizes of grains distributed near the impact area appear to be smaller than those in the far-field area, and most of the grain morphologies are also modified because of the repeated impact. However, the images can only present a rough result; therefore, it remains difficult to quantify the changes in grain size and a deeper investigation is necessary.

To quantify the grain size, lineal intercept approach as suggested by ASTM [19] was applied based on the sample metallographs as is shown in Figure 5. The grain size can be evaluated as follows: 


$$
d=\frac{l}{M \cdot P},
$$

where $d$ is the grain size, $l$ is the length of test line, $M$ is the image magnification, and $P$ is the number of intercepts where a test line is cut by a grain boundary. Details of the counting of intercepts may be found elsewhere [19]. Figure 9 shows an example of the lineal intercept approach. The grain size of each part was determined by three images near the reference point, and each image needed three test lines from which to count the intercepts. In the calculation, the image magnification $M$ was ignored because the image is life-size.

The results are listed in Table 2, and the equivalent grain size $d_{\text {eq }}$ is also given as

$$
d_{\mathrm{eq}}=\sqrt{d_{\mathrm{h}} \cdot d_{\mathrm{v}}}
$$

where $d_{\mathrm{h}}$ and $d_{\mathrm{v}}$ are the horizontal and vertical grain size, respectively.

According to the equivalent grain size $d_{\text {eq }}$ in Table 2, it is verified that the grain size near the impact surface is smaller than that in the far-field on each reference surface, which agrees well with earlier observations. As the Hall-Petch relationship [20] predicted, as provided in Equation (13), the material hardness will be increased with the decrease of grain size, resulting in strain-hardening effect.

$$
H=H_{0}+Q d^{-1 / 2} \text {, }
$$

where $H$ is the material hardness, and $H_{0}$ and $Q$ are the material constants.

In addition, the ratio of $d_{\mathrm{h}}$ versus $d_{\mathrm{v}}$ is also defined as the grain shape factor, and it is found that the grain will be further stretched, near the impact area, due to the large plastic deformation.

3.4. Degree of Hardenability. Since the depth indicator can only detect the V.CPD from the surface, the inside plastic deformation has to be evaluated, separately, by determining other relative material properties. The hardness variation, as predicted by the Hall-Petch relationship, provides an alternative approach via evaluation of the hardening degree $\Delta H$

$$
\Delta H=H_{\text {if }}-H_{\mathrm{i}},
$$

where $H_{\text {if }}$ is the hardness after deformation and $H_{\mathrm{i}}$ is the initial hardness.

Figure 10 shows the measured result: near the impact surface, an increase in hardening degree is obtained, indicating that large plastic deformation occurs near the impact surface; however, the degree of hardening decreases sharply through the sample thickness. Figure 10 also shows that the depth of the zone affected by repeated impact is about $3 \mathrm{~mm}$, i.e., the material was hardened up to this depth, and this could be the limit of the plastic deformation region, as well as of the residual stress field. Moreover, when comparing the degree of hardening at reference surfaces near the impact region, surface I

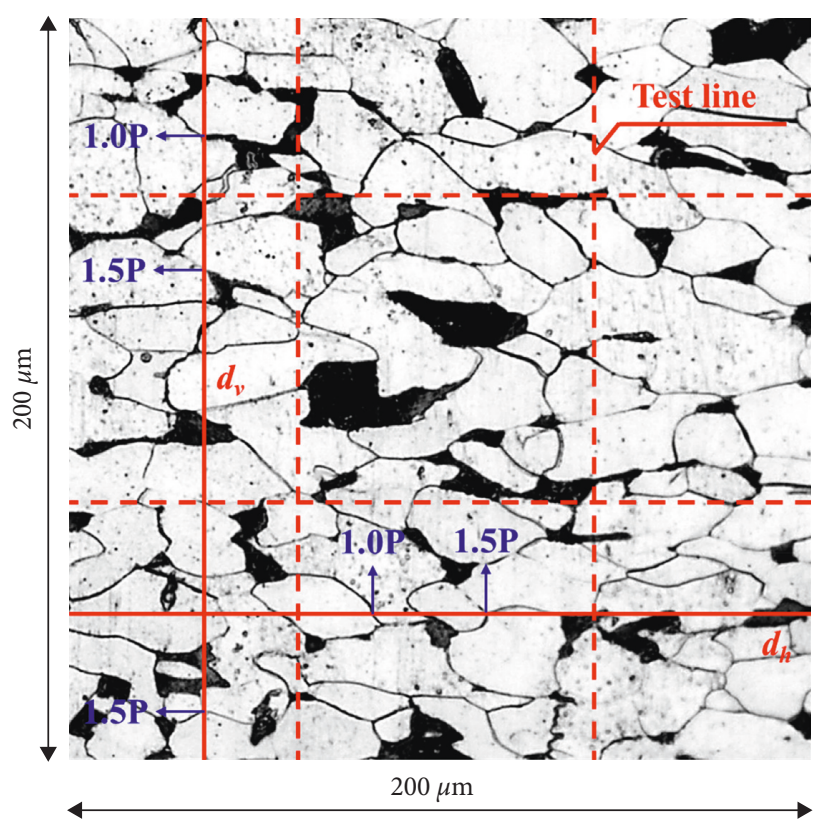

FIGURE 9: Lineal intercept approach.

TABLe 2: Grain size (unite: $\mu \mathrm{m}$ ).

\begin{tabular}{lcccc}
\hline Image number & $d_{\mathrm{h}}$ & $d_{\mathrm{v}}$ & $d_{\mathrm{eq}}$ & $d_{\mathrm{h}} / d_{\mathrm{v}}$ \\
\hline I-1 & 11.76 & 8.97 & 10.27 & 1.311 \\
I-2 & 13.04 & 10.62 & 11.77 & 1.228 \\
II-1 & 16.90 & 9.84 & 12.90 & 1.717 \\
II-2 & 16.00 & 12.77 & 14.29 & 1.253 \\
III-1 & 12.77 & 10.34 & 11.49 & 1.235 \\
III-2 & 14.63 & 11.88 & 13.18 & 1.231 \\
\hline
\end{tabular}

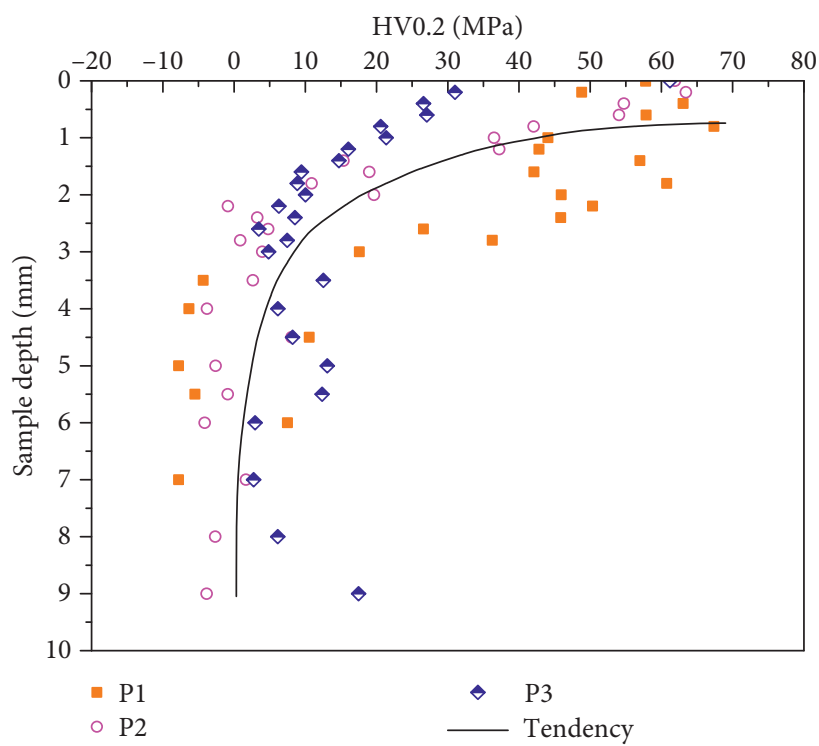

Figure 10: Hardening degree.

seems a slightly larger than other two, and surface III is the smallest. These differences might predict the relationship of plastic deformation at reference surface: I > II > III. 


\section{Simulation on the Plastic Deformation Behavior}

4.1. Finite-Element Model. The finite-element model was established by using the commercial software Abaqus with the same size of samples, as shown in Figure 11. A cube-shaped chisel with $5 \mathrm{~mm}$ edge length was established and defined as the rigid body. The bottom of the chisel corner was rounded with a radius of $0.5 \mathrm{~mm}$ to avoid element distortion. Since the plastic deformation is the main factor of concern, $1 \mathrm{~mm}$ mesh size was applied to the whole target material, with element type C3D8R selected. An elastoplastic analysis was carried out with the material's Young modulus $206 \mathrm{GPa}$, Poisson ratio 0.3 , and density $7900 \mathrm{Kg} / \mathrm{m}^{3}$. The Johnson-Cook model [21] was applied to deal with the dynamic yield strength at high strain rates. Two different models were considered, named M-Edge and M-Mid, according to experimental design. The impact depth was controlled to about $0.3 \mathrm{~mm}$, by adjusting the impact speed. For the sake of calculation efficiency, a single high-speed impact was applied instead of repeated impacts, because the final deformation was the main factor of concern here.

4.2. Transverse Deformation. Figure 12 shows a comparison of H.CPD in two directions: H.CPD in the $X$ direction (Figure 12(a)) on cracked surface, after impact, is about 3 times larger than that without crack, while it is similar in the $Z$ direction (Figure 12(b)). It gradually decreases through the thickness, becoming almost the same far from the impact surface. The curve of H.CPD in the $X$ direction for M-edge model seems similar to that indicated by the distribution of the degree of material hardening (Figure 10). Therefore, when applying the repeated impact after the onset of cracking, the value of H.CPD on the cracked surface plays a major role in affecting the local deformation, resulting in the contact between fracture surfaces.

The relationship between V.CPD (denoted by $S$ ) and $\mathrm{H}$. CPD (denoted by $L$ ) was then established by changing the impact speed, as shown in Figure 13. A one-to-one linear relation is then obtained, and the data measured from the experiment also supports this relation. In addition, when a large plastic deformation occurs due to impact, the ratio between $L$ and $S$ gradually departs from the fitted curve, which might result in calculation error; however, based on the prediction of Equation (3) and relation in Figure 12(a), the limit to V.CPD is about $1.2 \mathrm{~mm}$ subjected to $5 \mathrm{~mm} \times 5 \mathrm{~mm}$ chisel size. Therefore, the linear relationship remains applicable for prediction of the transverse deformation on cracked surface. Moreover, with the help of this relationship, the contact between fracture surfaces can be predicted by calculating V.CPD by equations based on the device parameters used, and some impact parameters can be adjusted before treatment, to fit actual demand.

4.3. Influence of Chisel Tip Parameters. During actual crackclosure treatment, the device parameters remain unchanged, because the air compressor and impact air tool are rarely replaced. The parameters of the chisel tip, such as the size, shape, and radius, might be the main factors affecting the crack-closure effect, because these parameters are not constant due to different needs by operators, and they will also change with the wear of tool and material. Therefore, the influences thereof on the relationship mentioned above under different parameters of the chisel tip were further discussed on the basis of the output from the finite-element method.

The comparison was carried out under the same impact energy, and total three cases were considered. Case one considers the size effect under same chisel mass by changing the chisel material density, so each chisel kinetic energy $\left(0.5 m v^{2}\right)$ was the same. The case one approximately simulated the actual treatment, because the influence of original chisel mass when changing the chisel tip size was so small as to be ignored. Case two discussed the shape effect under same chisel tip size, simulating the possible situation when other shapes were used rather than a square shape. Case three compares with the effect of chisel tip radius (taking the value of $0-1 \mathrm{~mm}$, with an interval of $0.25 \mathrm{~mm}$ ) due to the tool and material wear, and only one side of the corner is round because of the actual operation.

The comparative results are shown in Figure 14, and it can be seen clearly that most of the data are well distributed within the range of $\pm 10 \%$ error, indicating the linear relationship between H.CPD and V.CPD is still available under different parameters of chisel tip. The conclusion obtained from Figure 13 was thus verified. Besides, by analysing each case, it is also found that reducing of chisel tip size will improve the plastic deformation, which is very useful to high-strength steel, and the shape has less influence since the data distribute relatively centralised.

\section{Conclusions}

The plastic deformation of cracked surface under multiple impacts was investigated in the paper. A numerical model illustrating the relation between plastic deformation and impact process was established, as well as the relationship between impact depths V.CPD and H.CPD. The influence on material properties was discovered via metallographic analysis and microhardness testing. The following conclusions can be drawn from this study:

(1) The established numerical model can predict the amount of plastic deformation (impact depth) experienced during repeated impact, considering actual impact device parameters.

(2) The material grain size near the impact surface becomes smaller due to repeated impact. Strain hardening was assessed by comparing the degree of hardening through sample thickness, and the material will be hardened only within the small range of the affected zone.

(3) When the crack occurs, the cracking surface will cause large plastic deformation, more so than in other directions under repeated impact, contributing to the contact of fracture surface. 


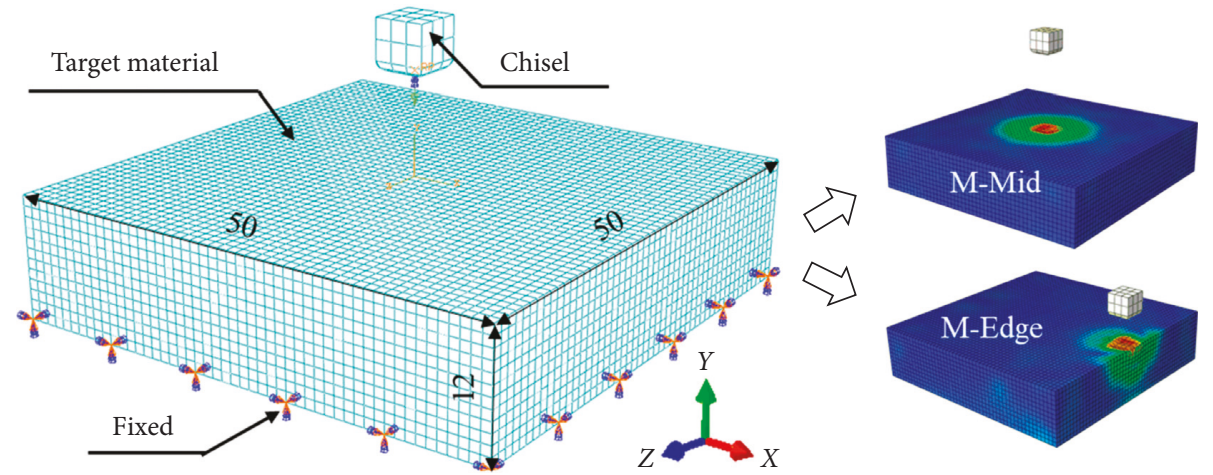

Figure 11: Finite-element model.

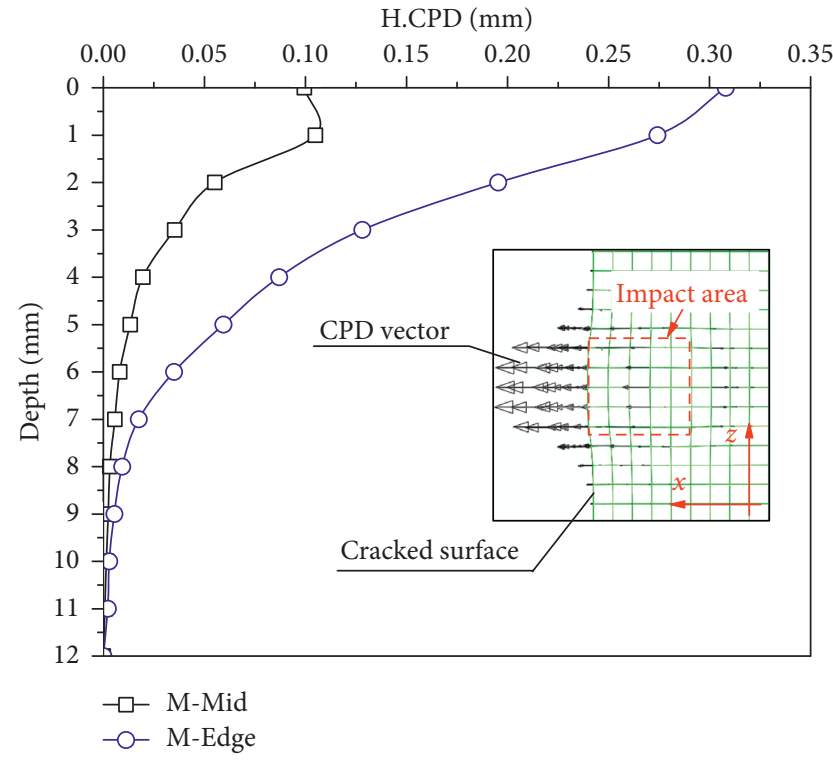

(a)

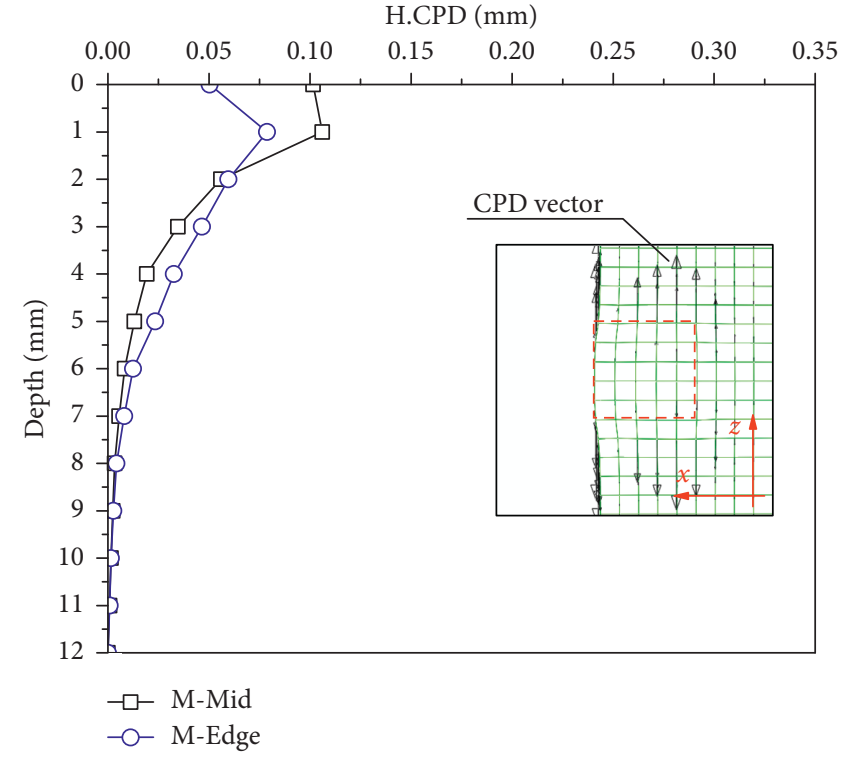

(b)

Figure 12: H.CPDs comparing. (a) $X$ direction. (b) $Z$ direction.

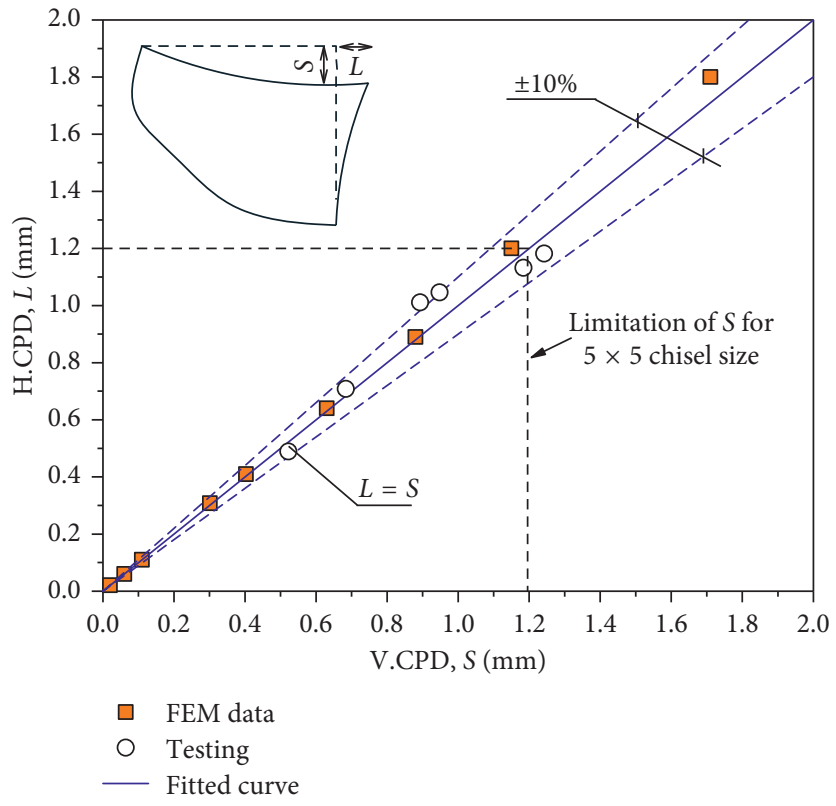

FIgURE 13: Relationship between V.CPD and H.CPD. 


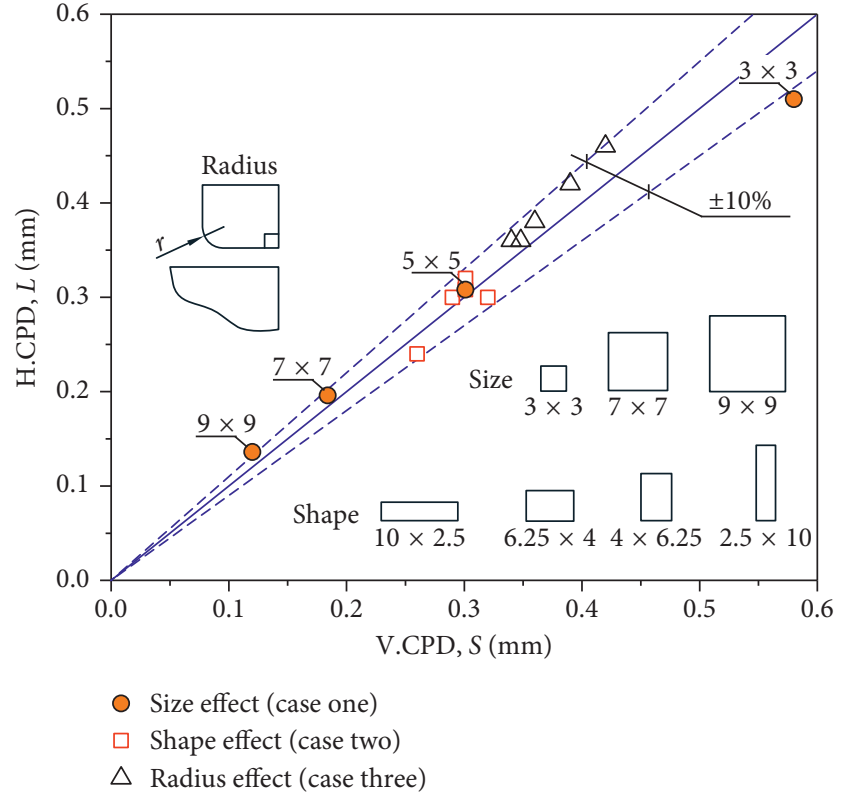

FIGURE 14: Influence of chisel tip parameters.

(4) A one-to-one linear relation between impact depth and horizontal deformation was obtained; therefore, the crack-closure behavior can be predicted with the help of this relationship and the associated numerical model.

\section{Data Availability}

The authors declare that all data are fully available without restriction. And readers can access the data from the corresponding author.

\section{Conflicts of Interest}

The authors declare that there are no conflicts of interest regarding the publication of this paper.

\section{Acknowledgments}

The research reported herein has been conducted as part of the research projects granted by the National Natural Science Foundation of China (51678216 and 51478163) and the Fundamental Research Funds for the Central Universities (2018B41614). The assistances are gratefully acknowledged.

\section{References}

[1] W. Schütz, "A history of fatigue," Engineering Fracture Mechanics, vol. 54, no. 2, pp. 263-300, 1996.

[2] Q. H. Zhang, Y. Z. Bu, and Q. Li, "Review on fatigue problems of orthotropic steel bridge deck," China Journal of Highway and Transport, vol. 30, no. 3, pp. 14-30, 2017.

[3] M. H. Kolstein, Fatigue Classification of Welded Joints in Orthotropic Steel Bridge Decks, Delft University of Technology, Delft, Netherlands, 2007.
[4] A. Murdani, C. Makabe, A. Saimoto, and R. Kondou, "A crack-growth arresting technique in aluminum alloy," Engineering Failure Analysis, vol. 15, no. 4, pp. 302-310, 2008.

[5] C. Miki, M. T. Hanji, and M. K. Tokunaga, "Weld repair for fatigue-cracked joints in steel bridges by applying low temperature transformation welding wire," Welding in the World, vol. 56, no. 3-4, pp. 40-50, 2013.

[6] J. W. Fisher and R. J. Dexter, "Field experience with repair of fatigue cracks," in Proceedings of International Conference on Fatigue, pp. 9-10, Germain en Laye, France, May 1994.

[7] C. M. Branco, V. Infante, and R. Baptista, "Fatigue behavior of welded joints with cracks repaired by hammer peening," Fatigue Fracture of Engineering Materials and Structures, vol. 27, no. 9, pp. 785-798, 2004.

[8] K. Yamada, T. Ishikawa, T. Kakiichi, and H. Li, "Development of elongation technique of fatigue life by closing fatigue crack," in Proceedings of Annual Conference of the Chubu Branch of JSCE, vol. 5, pp. 9-10, Nagano, Japan, 2009.

[9] K. Yamada, T. Kakiichi, and T. Ishikawa, "Extending fatigue life of cracked welded joint by impact crack closure retrofit treatment," in IIW Document XIII-2289 r1-09, International Institute of Welding, Singapore, 2009.

[10] K. Yamada, T. Ishikawa, T. Kakiichi et al., "Rehabilitation and improvement of fatigue life of welded joints by ICR treatment," Advanced Steel Construction, vol. 11, no. 3, pp. 294304, 2015.

[11] T. Ishikawa, K. Yamada, T. Kakiichi, and H. Li, "Extending fatigue life of cracked out-of-plane gusset by ICR treatment," Structural Engineering/Earthquake Engineering, vol. 28, no. 1, pp. 21-28, 2011.

[12] Z.Y. YuanZhou, B. H. Ji, Z. Q. Fu, and H. B. Ge, "Local stress variation in welded joints by ICR treatment," Journal of Constructional Steel Research, vol. 120, pp. 45-51, 2016.

[13] Z. Y. YuanZhou, B. H. Ji, Z. Q. Fu, and H. B. Ge, "Fatigue performance of cracked rib-deck welded joint retrofitted by ICR technique," International Journal of Steel Structures, vol. 16, no. 3, pp. 735-742, 2016.

[14] Z. Y. YuanZhou, B. H. Ji, Z. Q. Fu, and T. Sun, "Retarding effects on crack propagation by closing crack surface using ICR treatment," Journal of Constructional Steel Research, vol. 143, pp. 11-17, 2018.

[15] E. L. Layland, "How metals perform under repeated impact," Materials and Methods, vol. 44, p. 104, 1956.

[16] N. Brown and K. F. Lukens, "Microstrain in polycrystalline metals," Acta Metallurgica, vol. 9, no. 2, pp. 106-111, 1961.

[17] R. Seifried, W. Schiehlen, and P. Eberhard, "Numerical and experimental evaluation of the coefficient of restitution for repeated impacts," International Journal of Impact Engineering, vol. 32, no. 1-4, pp. 508-524, 2005.

[18] I. Argatov, M. Kachanov, and G. Mishuris, "On the concept of "far points" in hertz contact problems," International Journal of Engineering Science, vol. 113, pp. 20-36, 2017.

[19] ASTM E112-13, Standard Test Methods for Determining Average Grain Size, ASTM International, Conshohocken, PA, USA, 2013.

[20] Z. Yanushkevich, S. V. Dobatkin, A. Belyakov, and R. Kaibyshev, "Hall-Petch relationship for austenitic stainless steels processed by large strain warm rolling," Acta Materialia, vol. 136, pp. 39-48, 2017.

[21] A. Shokry, "A modified Johnson-Cook model for flow behavior of alloy $800 \mathrm{~h}$ at intermediate strain rates and high temperatures," Journal of Materials Engineering and Performance, vol. 26, no. 12, pp. 5723-5730, 2017. 


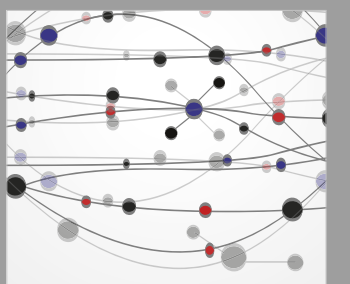

The Scientific World Journal
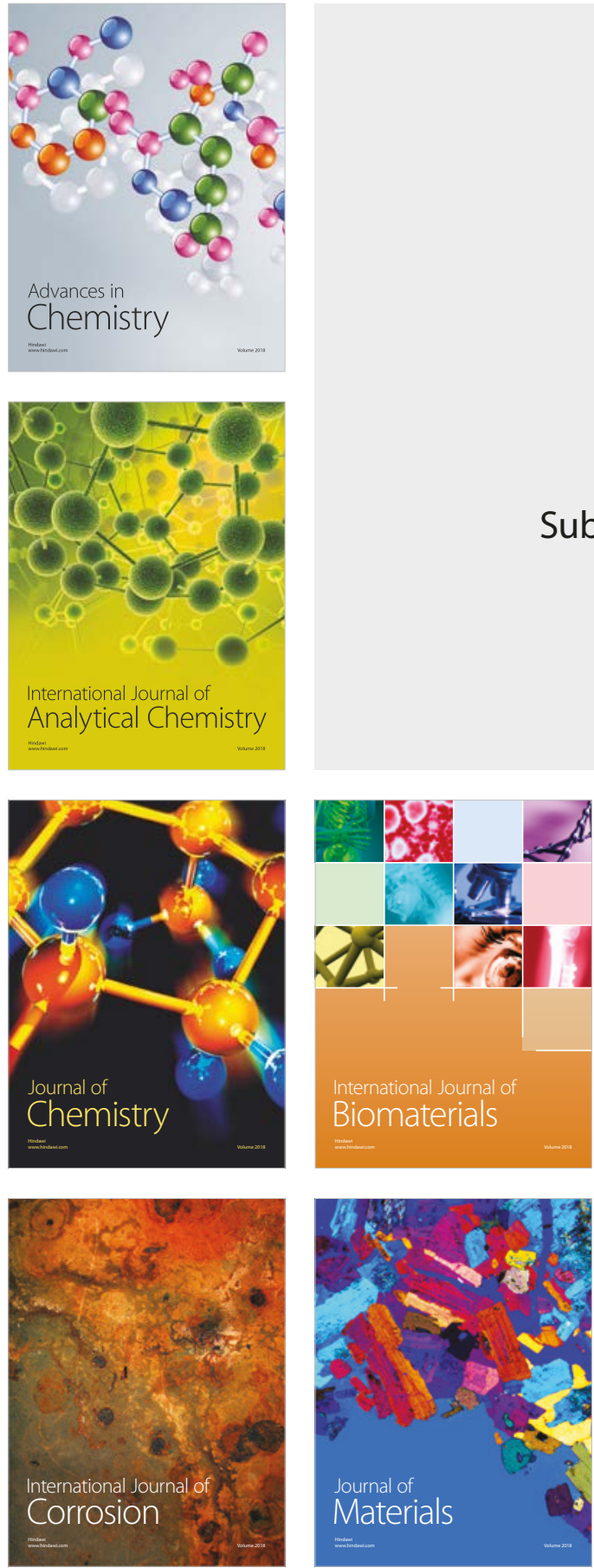

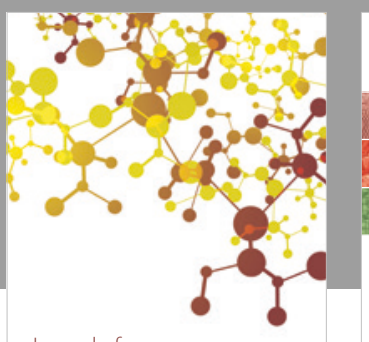

Journal of

Applied Chemistry
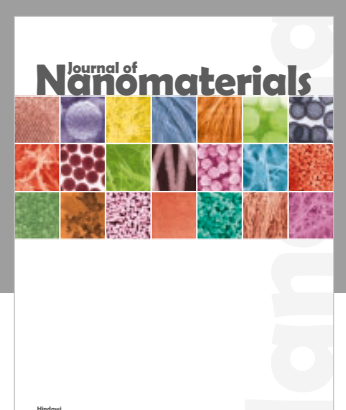

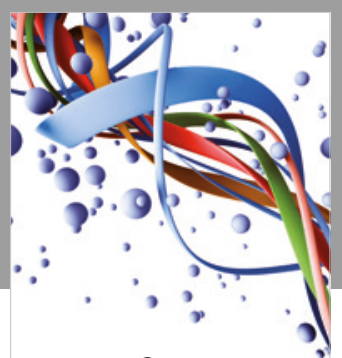

Scientifica

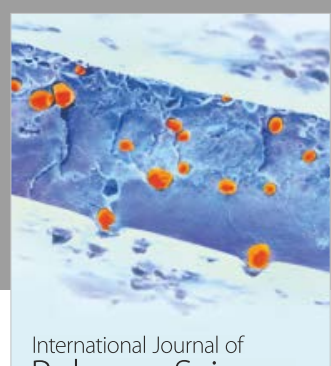

Polymer Science

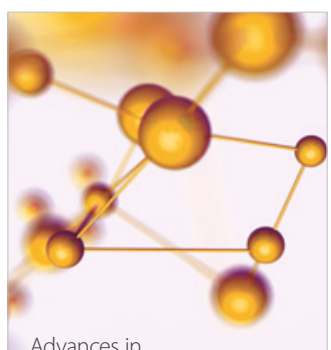

Physical Chemistry
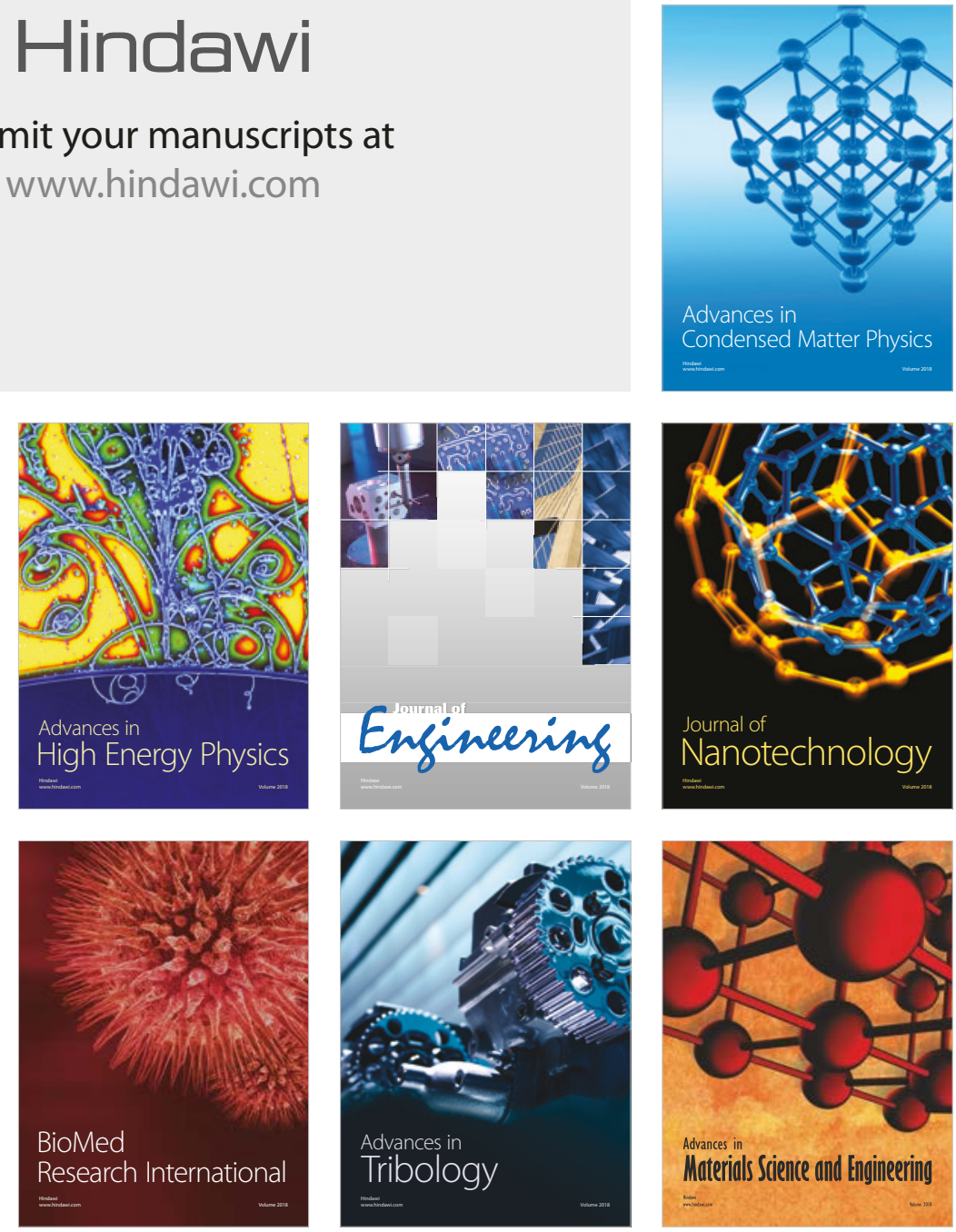